\title{
Approximating strongly correlated wave functions with correlator product states
}

\author{
Hitesh J. Changlani, ${ }^{1,2}$ Jesse M. Kinder, ${ }^{1}$ C. J. Umrigar, ${ }^{2}$ and Garnet Kin-Lic Chan ${ }^{1, *}$ \\ ${ }^{1}$ Department of Chemistry and Chemical Biology, Cornell University, Ithaca, New York 14853, USA \\ ${ }^{2}$ Department of Physics, Cornell University, Ithaca, New York 14853, USA \\ (Received 26 July 2009; revised manuscript received 28 October 2009; published 22 December 2009)
}

\begin{abstract}
We describe correlator product states, a class of numerically efficient many-body wave functions to describe strongly correlated wave functions in any dimension. Correlator product states introduce direct correlations between physical degrees of freedom in a simple way, yet provide the flexibility to describe a wide variety of systems. We show that many interesting wave functions can be mapped exactly onto correlator product states, including Laughlin's quantum Hall wave function, Kitaev's toric code states, and Huse and Elser's frustrated spin states. We also outline the relationship between correlator product states and other common families of variational wave functions such as matrix product states, tensor product states, and resonating valence-bond states. Variational calculations for the Heisenberg and spinless Hubbard models demonstrate the promise of correlator product states for describing both two-dimensional and fermion correlations. Even in onedimensional systems, correlator product states are competitive with matrix product states for a fixed number of variational parameters.
\end{abstract}

DOI: $10.1103 /$ PhysRevB.80.245116

PACS number(s): 71.15. $-\mathrm{m}, 71.27 .+\mathrm{a}$

\section{INTRODUCTION}

How can one efficiently approximate an eigenstate of a strongly correlated quantum system? In one-dimensional (1D) systems, the density-matrix renormalization group (DMRG) provides a powerful and systematic numerical approach. ${ }^{1,2}$ However, the accuracy of the DMRG in two or more dimensions is limited by the one-dimensional encoding of correlations in the matrix product states (MPS) that form the variational basis of the DMRG. ${ }^{2}$ Generalizations of MPS to higher dimensions-tensor network or tensor product states (TPS) (Refs. 3-8) - have been introduced recently, but these engender considerable computational complexity (which does not arise with MPS). This has made it difficult to practically extend the success and accuracy of the DMRG to higher dimensions.

In this paper we examine a different class of quantum states: correlator product states (CPS). Unlike MPS and TPS, which introduce auxiliary degrees of freedom to generate correlations between physical degrees of freedom, CPS correlate the physical degrees of freedom explicitly. The CPS form has been rediscovered many times, ${ }^{9-11}$ but the potential of CPS as an alternative to MPS/TPS for systematically approximating strongly correlated problems remains largely unexplored. Here we take up this possibility. CPS share many of the local properties of MPS/TPS but appear more suitable for practical calculations in more than one dimension as well as for fermion systems. To establish the potential of CPS, we analyze the relation between CPS and common families of analytic and numerical trial wave functions. We then discuss the most important properties of CPS: they permit efficient evaluation of observables and efficient optimization. Finally, we present variational Monte Carlo calculations for both spin and fermion systems. Our CPS results compare favorably with calculations using other variational wave functions that contain a similar number of variational parameters.

Note: As this paper was completed we were informed of recent work by Isaev et al. ${ }^{12}$ on hierarchical mean-field theory and by Mezzacapo et al. ${ }^{13}$ on entangled plaquette states as well as earlier work on string-bond states. ${ }^{14}$ All these studies consider wave functions similar to CPS and share many of our own objectives. However, while our current efforts are related, especially to Ref. 13, we focus on aspects of CPS not addressed in these other works, such as the relationship with well-known analytical and numerical wave functions, and we consider different physical problems, such as fermion simulations. Thus we regard our work as complementary rather than overlapping.

\section{CORRELATOR PRODUCT STATES}

Consider a set of quantum degrees of freedom $Q$ $\equiv\left\{q_{1}, q_{2}, \ldots, q_{L}\right\}$ on a lattice with $L$ sites in one or more dimensions. Each $q_{i}$ might represent a spin $S=1 / 2$ degree of freedom, where $q \in\{\uparrow, \downarrow\}$, or a fermion degree of freedom, in which case $q \in\{0,1\}$. An arbitrary quantum state can be expanded over all configurations as

$$
|\Psi\rangle=\sum_{\{q\}} \Psi^{q_{1} q_{2} \ldots q_{L}}\left|q_{1} q_{2} \ldots q_{L}\right\rangle .
$$

A general quantum wave function requires an exponential number of parameters - one for each configuration. One way to reduce the complexity of the problem is to impose some structure on the coefficients $\Psi(Q)$. CPS are one example of this approach.

CPS are obtained by associating variational degrees of freedom directly with correlations between groups of sites. For example, in the nearest-neighbor two-site CPS, a correlator is associated with each neighboring pair of sites

$$
|\Psi\rangle=\sum_{\{q\}} \prod_{\langle i j\rangle} C^{q_{i} q_{j}}\left|q_{1}, \ldots, q_{L}\right\rangle,
$$

where $\langle i j\rangle$ denote nearest neighbors. The coefficients in Eq. (1) are given by products of correlator coefficients. For ex- 

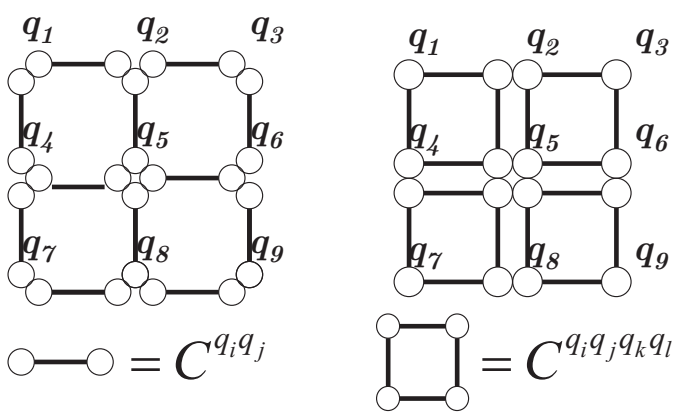

FIG. 1. Nearest-neighbor two-site and $2 \times 2$ plaquette CPS on a 2D lattice. The CPS weight for a given quantum configuration $\left|q_{1} q_{2} \ldots q_{L}\right\rangle$ is obtained by multiplying correlator coefficients together as in Eq. (2).

ample, in a one-dimensional lattice, the amplitude of a configuration is

$$
\Psi(Q)=C^{q_{1} q_{2}} C^{q_{2} q_{3}} C^{q_{3} q_{4}} \ldots C^{q_{L-1} q_{L}} .
$$

Equation (2) can be extended to higher dimensions simply by associating correlators with (overlapping) bonds on the lattice (Fig. 1). The nearest-neighbor two-site CPS is an extremely simple CPS. Longer range correlations can be introduced by removing the nearest-neighbor restriction on pair correlations or by including explicit correlations among more sites with correlators such as $C^{q_{1} q_{2} q_{3}}$. It is clear that CPS provide a complete basis: in the limit of $L$-site correlators, the CPS amplitudes are precisely the coefficients of Eq. (1).

When there is a global constraint on the total spin $S$ or particle number $N$ we can use projected CPS wave functions. For example, for fixed particle number, the $N$-projected nearest-neighbor two-site CPS is

$$
|\Psi\rangle=\sum_{\{q\}} \sum_{\langle i j\rangle} C^{q_{i} q_{j}} \hat{P}_{N}\left|q_{1}, \ldots, q_{L}\right\rangle,
$$

where $\hat{P}_{N}$ ensures that $\Sigma_{i} q_{i}=N$. Such projections do not introduce any complications in working with CPS and may be included in both deterministic and stochastic calculations without difficulty.

It is sometimes useful to write the CPS in a different form. Each correlator element $C^{q_{i} q_{j}}$ can be viewed as the matrix element of a correlator operator $\hat{C}^{i j}$ that is diagonal in the quantum basis $\left\{\left|q_{i} q_{j}\right\rangle\right\}$ :

$$
\left\langle q_{i} q_{j}\left|\hat{C}^{i j}\right| q_{i}^{\prime} q_{j}^{\prime}\right\rangle=\delta_{q_{i} q_{i}^{\prime}} \delta_{q_{j} q_{j}^{\prime}} C^{q_{i} q_{j}}
$$

The CPS wave function is obtained by acting a string of commuting correlator operators on a reference state $|\Phi\rangle$. For example, a two-site CPS may be written as

$$
|\Psi\rangle=\prod_{i>j} \hat{C}^{i j}|\Phi\rangle .
$$

When there are no constraints, the reference state is taken to be an equally weighted sum over all quantum configurations: $|\Phi\rangle=\Sigma_{\{q\}}\left|q_{1} q_{2} \ldots q_{L}\right\rangle$; otherwise, $|\Phi\rangle$ is projected to satisfy the constraint. For example, if particle number is fixed, $\left|\Phi_{N}\right\rangle$ is an equally weighted sum over all quantum configurations with particle number $N$,

$$
\left|\Phi_{N}\right\rangle=\sum_{\{q\}} \hat{P}_{N}\left|q_{1} q_{2} \ldots q_{L}\right\rangle .
$$

Note that both projectors and correlators are diagonal operators in the Hilbert space and commute with one another: this means that the projection can be applied directly to the reference state and this simplifies numerical algorithms using CPS. The operator representation is also useful when considering extensions to the CPS form such as alternative reference states.

\section{CONNECTION TO OTHER WAVE FUNCTIONS}

Many strongly correlated quantum states can be represented exactly as correlator product states. CPS also have much in common with several classes of widely used variational wave functions: matrix product states, tensor product states, and resonating valence-bond states. In this section, we discuss the connections between these wave functions.

\section{A. Huse-Elser wave functions}

In their study of frustrated spin systems, Huse and Elser constructed states in which the quantum amplitudes $\Psi(Q)$ correspond to classical Boltzmann weights $\exp (-\beta E[Q] / 2)$ multiplied by a complex phase. ${ }^{10}$ The weights are derived from an effective classical Hamiltonian $\hat{H}^{c l}$. For example, in the case of pairwise correlations, $\hat{H}^{c l}=\Sigma_{i j} \hat{h}_{i j}^{c l}$ with $\hat{h}_{i j}^{c l}$ $=K_{i j} \hat{S}_{z}^{i} \hat{S}_{z}^{j}$. The corresponding wave function can be represented as a two-site CPS with $\hat{C}^{i j}=\exp \left(-\beta \hat{h}_{i j}^{c l} / 2+i \hat{\phi}_{i j}\right)$, where $\hat{\phi}_{i j}$ assigns a complex phase to the pair $i j$.

For the square and triangular Heisenberg lattices, Huse and Elser demonstrated that a very compact variational ground state could be obtained with a semianalytic threeparameter model for $\hat{H}^{c l}$ (containing up to three-site interactions) and an analytically determined phase. Although CPS can represent such highly constrained wave functions for symmetric systems, it can also serve as the foundation of a more general numerical method. By allowing correlators to vary freely and by considering hierarchies of larger correlated clusters, we can hope to construct rapidly converging approximations to arbitrary strongly correlated quantum states, as the DMRG does for one-dimensional quantum problems.

\section{B. Laughlin wave function}

In 1983, Laughlin proposed a variational wave function to explain the fractional quantum Hall effect. ${ }^{15}$ The Laughlin wave function describes a strongly interacting system with topological order. Like the Huse and Elser wave functions, Laughlin's wave function can be associated with the Boltzmann weights of an effective classical Hamiltonian and can be represented exactly as a correlator product state.

The Laughlin quantum Hall state at filling fraction $1 / \mathrm{m}$ can be written in first quantization as 


$$
\Psi\left(\mathbf{r}_{1}, \ldots, \mathbf{r}_{N}\right)=\prod_{\lambda<\mu}^{N}\left(z_{\lambda}-z_{\mu}\right)^{m} e^{-\alpha \Sigma_{\kappa}\left|z_{\kappa}\right|^{2}},
$$

where $z_{\lambda}$ is the (complex) coordinate of particle $\lambda$. (A Greek subscript indicates the coordinate of a particular electron. A Roman subscript indicates the coordinate of a lattice site.) Alternatively, the system can be mapped onto a discrete set of coordinates $z_{1}, \ldots, z_{L}$ with an associated set of occupation numbers $q_{1}, \ldots, q_{L}$. Then Eq. (8) can be exactly expressed as a two-site CPS in the occupation number representation

$$
\begin{gathered}
|\Psi\rangle=\sum_{\{q\}} \prod_{i} C_{1}^{q_{i}} \prod_{i<j} C_{2}^{q_{i} q_{j}} P_{N}\left|q_{1}, \ldots, q_{L}\right\rangle, \\
\mathbf{C}_{\mathbf{1}}=\left(\begin{array}{c}
1 \\
e^{-\alpha\left|z_{i}\right|^{2}}
\end{array}\right), \\
\mathbf{C}_{\mathbf{2}}=\left(\begin{array}{cc}
1 & 1 \\
1 & \left(z_{i}-z_{j}\right)^{m}
\end{array}\right) .
\end{gathered}
$$

The CPS wave function exactly reproduces the Laughlin wave function. It is, in some ways, more general than Eq. (8). The CPS form could be used to extend the Laughlin state beyond two-site correlators while maintaining antisymmetry of the state, or to find a better variational energy in open or disordered systems.

\section{Toric code}

Kitaev's toric code is another interesting quantum state with an exact CPS representation. Kitaev proposed the toric code as a model for topological quantum computing. The Hamiltonian is a sum of site and plaquette projectors on a square lattice with spins placed on the bonds. On a torus, the ground state of this Hamiltonian is fourfold degenerate with a gap to all other excitations. ${ }^{16}$ It is an example of a quantum system with topological order.

The ground state can be obtained from the zerotemperature Boltzmann weights of a classical Hamiltonian $\hat{H}_{\text {toric }}^{c l}=\Sigma_{\square_{i}} \hat{h}_{\square_{i}}$. The sum is over all plaquettes $\square_{i}$, and $\hat{h}_{\square_{i}}$ is a product of $\hat{S}_{z}$ operators associated with the spins on the edges of the plaquette. ${ }^{4}$ The amplitudes of the toric code wave function can be generated by a CPS with plaquette correlators

$$
C_{\square}^{i j k l}= \begin{cases}1 & \text { if } S_{z}^{i} S_{z}^{j} S_{z}^{k} S_{z}^{l}>0, \\ 0 & \text { if } S_{z}^{i} S_{z}^{j} S_{z}^{k} S_{z}^{l}<0 .\end{cases}
$$

The exact representation of the toric code and Laughlin's wave function demonstrates the ability of CPS to describe systems with topological order.

\section{MPS and TPS}

Correlator product states are conceptually related to matrix and tensor product states. All of these wave functions can easily express entanglement between local degrees of freedom. Nonetheless, CPS and MPS/TPS form different classes of quantum states and one is not a proper subset of the other.

A MPS is obtained by approximating the quantum amplitudes $\Psi(Q)$ in Eq. (1) as a product of matrices, one for each site on the lattice

$$
\Psi(Q)=\sum_{\{i\}} A_{i_{1} i_{2}}^{q_{1}} A_{i_{2} i_{3}}^{q_{2}} \ldots A_{i_{L} i_{1}}^{q_{L}}
$$

The "auxiliary" indices $\{i\}$ are contracted in a onedimensional pattern - a matrix product—and this gives rise to the low computational cost of working with MPS. However, the one-dimensional structure prevents MPS from efficiently describing correlations in higher dimensions. TPS extend MPS by approximating the amplitudes $\Psi(Q)$ by more general tensor contractions. Because of the more complicated contraction pattern, TPS can, in principle, describe higher dimensional correlations. ${ }^{4,6,7}$ Unlike MPS, the TPS contraction cannot be evaluated efficiently in general. This leads to the high computational cost of working with TPS.

To demonstrate the relationship between CPS and MPS/ TPS, we consider a simple example of a nearest-neighbor two-site CPS on a three-site lattice with periodic boundary conditions. These CPS amplitudes are

$$
\Psi^{q_{1} q_{2} q_{3}}=C^{q_{1} q_{2}} C^{q_{2} q_{3}} C^{q_{3} q_{1}} .
$$

Applying singular value decomposition to one of the correlators gives

$$
C^{q q^{\prime}}=\sum_{i} U_{i}^{q} \sigma_{i} V_{i}^{q^{\prime}}=\sum_{i} U_{i}^{q} W_{i}^{q^{\prime}}
$$

where we have absorbed the diagonal matrix $\sigma_{i}$ into $W_{i}^{q^{\prime}}$. With this decomposition, $|\Psi\rangle$ can be mapped to a MPS of auxiliary dimension 2 :

$$
\Psi^{q_{1} q_{2} q_{3}}=\sum_{\{i\}} U_{i_{1}}^{q_{1}}\left(W_{i_{1}}^{q_{2}} U_{i_{2}}^{q_{2}}\right)\left(W_{i_{2}}^{q_{3}} U_{i_{3}}^{q_{3}}\right) W_{i_{3}}^{q_{1}} .
$$

This is equivalent to Eq. (13) with $A_{i j}^{q}=W_{i}^{q} U_{j}^{q}$. The matrices of the resulting MPS (of dimension 2) have a restricted form. More complicated CPS (e.g., with three-site correlators) map to MPS with larger auxiliary dimension and more flexible forms for the matrices. (The dimension of the matrices grows exponentially with the range or number of sites in the correlator.) An arbitrary MPS cannot be mapped onto a CPS with less than the complete basis of $L$-site correlators. Conversely, a one-dimensional CPS with long-range correlators (such as the general two-site CPS used in the Laughlin state) can only be represented by a MPS with an auxiliary dimension that spans the full Hilbert space. These arguments can be extended to higher dimensions and similar conclusions hold for the mappings between CPS and TPS. For a given number of variational degrees of freedom, only a subset of CPS can be exactly written as MPS/TPS and vice versa.

While the correlators in the CPS have no auxiliary indices, they could be augmented by additional auxiliary indices. For example, string-bond states may be considered one-site correlators with a pair of auxiliary indices. ${ }^{14} n$-site correlators can be generalized in an analogous way. 
The concept of an area law is sometimes used in the analysis of wave functions. If the amount of entanglement between a system and its environment scales with the area of the boundary between the two, the system is said to obey an area law. Arguments from quantum-information theory suggest that wave functions that satisfy an area law can accurately describe systems (in any dimension) with a finite correlation length. ${ }^{17}$ (Some critical systems with long-range correlations also satisfy an area law, but others may violate the area law at zero temperature.)

MPS wave functions satisfy a one-dimensional area law and have a finite correlation length. (Long-range correlations can be reproduced over a finite range, but they will eventually decay exponentially.) TPS satisfy area laws in two or more dimensions. CPS with local correlators such as nearestneighbor pairs or plaquettes also satisfy an area law, making them promising candidates for systems with a finite correlation length. CPS with long-range correlators, such as those used in the Laughlin wave function, are not constrained by an area law and can describe even more entanglement between system and environment, obeying a volume law instead.

\section{E. RVB states}

Resonating valence-bond (RVB) states are widely used in strongly correlated quantum problems. ${ }^{18,19}$ A fermion RVB state can be written as a product of a Jastrow factor and a projected BCS wave function

$$
\left|\Psi_{\mathrm{RVB}}\right\rangle=e^{\Sigma_{i j} J_{i j} \hat{n}_{i} \hat{n}_{j}} \hat{P}_{N} e^{\Sigma_{i j} \lambda_{i j} a_{i}^{\dagger} a_{j}^{\dagger}}|\mathrm{vac}\rangle,
$$

where $J_{i j}$ and $\lambda_{i j}$ are commonly taken to be real.

There is a close relationship between CPS and RVB states. At half-filling, the $N$-projected two-site CPS can be expressed in the form of Eq. (17). Consider a dimer covering of the lattice. Let $\lambda_{i j}=1$ for each pair $i j$ that is connected by a dimer and $\lambda_{i j}=0$ otherwise. The corresponding projected BCS state is the CPS reference $\left|\Phi_{N}\right\rangle$ defined earlier

$$
\hat{P}_{N} e^{\Sigma_{i<j} a_{i}^{\dagger} a_{j}^{\dagger}}|\mathrm{vac}\rangle=\hat{P}_{N} \sum_{\{q\}}\left|q_{1} q_{2} \ldots q_{L}\right\rangle=\left|\Phi_{N}\right\rangle .
$$

If the Jastrow factor is allowed to become complex, then the two-site correlator $\hat{C}^{i j}$ is fully parameterized as

$$
\hat{C}^{i j}=\exp \left(J_{0}+J_{1}^{i} \hat{n}_{i}+J_{1}^{j} \hat{n}_{j}+J_{2}^{i j} \hat{n}_{i} \hat{n}_{j}\right)
$$

and the CPS and RVB wave functions are identical.

Despite the existence of a mapping between the two wave functions, the emphasis of the CPS parameterization is quite different from that of commonly studied RVB states. For fermion RVB wave functions where $J_{i j}$ is real, the Jastrow factor is positive and the nodes of the fermion wave function are those of the projected BCS state. In general, such a wave function cannot be exact. In contrast, the CPS wave function can modify the nodes of the reference wave function $\left|\Phi_{N}\right\rangle$ through the complex Jastrow factor. By using higher order correlators, the CPS state can therefore become exact. While the most flexible RVB/CPS form would combine a complex Jastrow factor with an arbitrary projected BCS reference, there are computational advantages to the simpler CPS reference, including the possibility to efficiently evaluate observables without the use of a stochastic algorithm. ${ }^{20}$

\section{COMPUTATIONAL COST OF CPS}

To be useful in practical calculations, a variational wave function must allow efficient evaluation of expectation values and optimization of its parameters. This combination of properties in matrix product states is responsible for the success of the density-matrix renormalization group. The expectation value of typical observables can be evaluated exactly in a time which is polynomial in the size of the system. Likewise, the amplitude of a given configuration can also be evaluated exactly in polynomial time. As shown in Eq. (13), the amplitude of a configuration is the trace of the product of $L$-independent $m \times m$ matrices, where $m$ is the dimension of the auxiliary indices $\{i\}$ and $L$ is the number of lattice sites. The cost for evaluating the amplitude is $\mathcal{O}\left(\mathrm{m}^{3} \mathrm{~L}\right)$.

Tensor product states generalize the structure of MPS to higher dimensions, but numerical efficiency is lost. In general, TPS amplitudes cannot be evaluated exactly in polynomial time! Additional renormalization procedures must be used while performing the contractions, which introduces an error that depends on the system size. For fermions, such errors can result in amplitudes or expectation values incompatible with a fermion wave function as well as a variational energy below the fermion ground state, a socalled $N$-representability problem. As a result, only certain classes of TPS are capable of efficient polynomial simulation.

Like MPS, correlator product states allow efficient, exact evaluation of wave-function amplitudes and expectation values. For example, the amplitudes of a pair CPS are $\Psi(Q)$ $=\prod_{i<j} C^{q_{i} q_{j}}$. The amplitude is a simple product of numbers. This is true for any CPS, and thus the complexity is proportional only to the number of correlators in the ansatz. This is manifestly polynomial in the system size. In general, evaluation of the amplitude with $n$-site correlators will require $\mathcal{O}(L)$ multiplications if the correlators act locally-e.g, nearest neighbors, plaquettes, etc.-and $\mathcal{O}\left(L^{n}\right)$ if there are no restrictions.

This property allows efficient Monte Carlo sampling of expectation values. (Deterministic algorithms can also be used but will be presented elsewhere. ${ }^{20}$ ) Moreover, constraints such as fixed particle number or total spin are easily handled within the Monte Carlo algorithm by limiting the Metropolis walk to states that satisfy these constraints. The expectation value of an operator is given by $\langle A\rangle$ $=\Sigma_{Q} P(Q) A(Q)$, where $P(Q)=|\Psi(Q)|^{2}$ and

$$
A(Q)=\sum_{Q^{\prime}}\left\langle Q|\hat{A}| Q^{\prime}\right\rangle \frac{\Psi\left(Q^{\prime}\right)}{\Psi(Q)} .
$$

The sum over $Q^{\prime}$ extends over only those $Q^{\prime}$ for which $\left\langle Q|\hat{A}| Q^{\prime}\right\rangle \neq 0$. As long as $\hat{A}$ is sparse in the chosen basis, its expectation value can be evaluated efficiently. If $|\Psi\rangle$ is local (e.g., nearest-neighbor pair CPS), a further simplification occurs for operators such as $a_{i}^{\dagger} a_{j}, a_{i}^{\dagger} a_{j}^{\dagger} a_{k} a_{l}$, or $\mathbf{S}_{i} \cdot \mathbf{S}_{j}$. For these 
TABLE I. Variational Monte Carlo energies (in units of $J$ ) using CPS for the $2 \mathrm{D} S=1 / 2$ Heisenberg model, including percent errors $(\Delta E)$. CPS[2] denotes nearest-neighbor two-site correlators and CPS $[n \times n]$ denotes plaquette correlators. The "exact" $6 \times 6$ and $8 \times 8$ energies are obtained from a stochastic series expansion MC calculation using ALPS (Ref. 21). Unlike matrix product states, correlator product states maintain good accuracy as the width is increased.

\begin{tabular}{|c|c|c|c|c|c|c|c|}
\hline Lattice & CPS[2] & $\begin{array}{l}\Delta E \\
(\%)\end{array}$ & $\mathrm{CPS}[2 \times 2]$ & $\begin{array}{l}\Delta E \\
(\%)\end{array}$ & $\mathrm{CPS}[3 \times 3]$ & $\begin{array}{l}\Delta E \\
(\%)\end{array}$ & Exact \\
\hline \multicolumn{8}{|c|}{ Periodic Boundary Conditions } \\
\hline $4 \times 4$ & $-11.057(1)$ & 1.5 & $-11.109(1)$ & 1.1 & $-11.2202(2)$ & 0.1 & -11.2285 \\
\hline $6 \times 6$ & $-23.816(3)$ & 2.6 & $-24.052(2)$ & 1.6 & $-24.313(2)$ & 0.5 & $-24.441(2)$ \\
\hline $8 \times 8$ & $-41.780(5)$ & 3.1 & $-42.338(4)$ & 1.8 & $-42.711(3)$ & 0.9 & $-43.105(3)$ \\
\hline \multicolumn{8}{|c|}{ Open Boundary Conditions } \\
\hline $4 \times 4$ & $-8.8960(5)$ & 3.2 & $-9.0574(4)$ & 1.4 & $-9.1481(2)$ & 0.5 & -9.1892 \\
\hline $6 \times 6$ & $-20.811(1)$ & 4.2 & $-21.176(1)$ & 2.5 & $-21.510(1)$ & 1.0 & $-21.727(2)$ \\
\hline $8 \times 8$ & $-37.846(3)$ & 4.5 & $-38.511(2)$ & 2.8 & $-39.109(2)$ & 1.3 & $-39.616(2)$ \\
\hline
\end{tabular}

operators, most of the factors in $\Psi(Q)$ and $\Psi\left(Q^{\prime}\right)$ are identical and cancel from the ratio so that the time required to evaluate the expectation value is independent of the system size and depends only on the number of Monte Carlo samples.

As with MPS and TPS, we can take advantage of the product structure of CPS when minimizing the variational energy and use an efficient local optimization or "sweep" algorithm. The energy is minimized with respect to one of the correlators while the others are held fixed, then repeated for each of the correlators in turn until the energy has converged. This algorithm is described in more detail in the next section.

\section{SPIN AND FERMION SIMULATIONS}

We have implemented a pilot variational Monte Carlo code to optimize general spin and fermion CPS wave functions. In Tables I and II we present results for two models of interacting spins and fermions: (i) the two-dimensional (2D) square Heisenberg model defined by the Hamiltonian

$$
H=J \sum_{\langle i j\rangle} \mathbf{S}_{i} \cdot \mathbf{S}_{j}
$$

and (ii) a 1D spinless Hubbard model at half-filling. This model is defined by the Hamiltonian

$$
H=\sum_{\langle i j\rangle}-t\left(a_{i}^{\dagger} a_{j}+a_{j}^{\dagger} a_{i}\right)+U n_{i} n_{j} .
$$

Each site can only be occupied or unoccupied, and the energy $U$ is the cost of placing two fermions on neighboring sites. We studied periodic and open boundary conditions for both the Heisenberg and Hubbard models.

\section{A. Optimization method}

We optimize the correlators by minimizing the variational energy with a sweep algorithm. At each step of each sweep, a target correlator is updated while the other correlators are fixed. Because the wave function $|\Psi\rangle$ is linear in the target correlator coefficients, the derivatives of $|\Psi\rangle$ with respect to these coefficients define a vector space for the optimization. For instance, if the target correlator has elements $C^{\mu}$, then the vector space is generated by the basis $\left|\widetilde{\Psi}_{\mu}\right\rangle$, where

$$
\left|\widetilde{\Psi}_{\mu}\right\rangle=\frac{\partial|\Psi\rangle}{\partial C^{\mu}} .
$$

Any vector in this space defines a CPS wave function: $\mathbf{x}$ corresponds to the wave function $|\Psi(\mathbf{x})\rangle=\Sigma_{\mu} x^{\mu}\left|\widetilde{\Psi}_{\mu}\right\rangle$.

It is convenient to work in a slightly different basis in which one vector $\mathbf{x}_{0}$ corresponds to the current value of the target correlator and the other vectors $\mathbf{x}_{i}$ are orthogonal to $\mathbf{x}_{0}$ (but not necessarily to each other). The updated target correlator will be a linear combination of the $\mathbf{x}_{\alpha}$, where $\alpha$ $\in\{0, i\}$.

We construct the Hamiltonian $\mathcal{H}_{\alpha \beta}$ and the overlap matrix $\mathcal{S}_{\alpha \beta}$ in this space

$$
\begin{aligned}
\mathcal{H}_{\alpha \beta} & =\left\langle\Psi\left(\mathbf{x}_{\alpha}\right)|\hat{H}| \Psi\left(\mathbf{x}_{\beta}\right)\right\rangle, \\
\mathcal{S}_{\alpha \beta} & =\left\langle\Psi\left(\mathbf{x}_{\alpha}\right) \mid \Psi\left(\mathbf{x}_{\beta}\right)\right\rangle,
\end{aligned}
$$

where $\hat{H}$ is the model Hamiltonian of Eq. (21) or (22). We then solve the generalized eigenvalue problem

$$
\mathcal{H} \cdot \mathbf{C}=\lambda \mathcal{S} \cdot \mathbf{C}
$$

where $\mathbf{C}$ is a linear combination of the $\mathbf{x}_{\alpha}$. The eigenvector with the lowest eigenvalue defines the optimal target correlator coefficients $\widetilde{C}^{\mu}$ that give the lowest energy when all other correlators are fixed. We sweep over all of the correlators one at a time until the energy stops decreasing.

This defines a general sweep algorithm for optimizing CPS. However to converge the sweeps when the Hamiltonian and overlap matrix are constructed via Monte Carlo sampling it is very important to minimize the stochastic error. Nightingale and Melik-Alaverdian, ${ }^{22}$ and Toulouse and Umrigar, ${ }^{23}$ defined efficient estimators for variational Monte Carlo optimization, and we have used these to construct $\mathcal{H}$ and $\mathcal{S}$. For numerical stability, it is important to monitor the 
TABLE II. Variational Monte Carlo energies (in units of $t$ ) for the $L$-site 1D spinless Hubbard model with repulsion $U$ using periodic and open boundary conditions, including percent errors $(\Delta E)$. CPS $[n]$ denotes $n$-site correlators; DMRG $[m]$ denotes a DMRG calculation with $m$ renormalized states. Since CPS and DMRG calculations are not directly comparable in terms of complexity, the approximate number of degrees of freedom per site (dof) is listed in the bottom row. (The numbers are exact in the limit of an infinite lattice.) Encouragingly, CPS is competitive with MPS for a comparable number of variational parameters. Exact energies are from $m=500$ DMRG calculations.

\begin{tabular}{|c|c|c|c|c|c|c|c|c|c|c|}
\hline$L$ & $U$ & CPS[3] & $\begin{array}{l}\Delta E \\
(\%)\end{array}$ & DMRG[3] & $\begin{array}{l}\Delta E \\
(\%)\end{array}$ & CPS[4] & $\begin{array}{l}\Delta E \\
(\%)\end{array}$ & DMRG[4] & $\begin{array}{l}\Delta E \\
(\%)\end{array}$ & Exact \\
\hline \multicolumn{11}{|c|}{ Periodic Boundary Conditions } \\
\hline 12 & 0 & $-7.052(1)$ & 5.5 & -7.165 & 4.0 & $-7.213(1)$ & 3.4 & -7.313 & 2.0 & -7.464 \\
\hline 12 & 4 & $-2.692(2)$ & 4.1 & -2.577 & 8.2 & $-2.756(1)$ & 1.8 & -2.725 & 2.9 & -2.807 \\
\hline 12 & 8 & $-1.461(1)$ & 1.1 & -1.430 & 3.1 & $-1.474(1)$ & 0.2 & -1.462 & 1.0 & -1.477 \\
\hline 24 & 0 & $-14.432(2)$ & 5.0 & -14.608 & 3.8 & $-14.714(2)$ & 3.2 & -14.832 & 2.4 & -15.192 \\
\hline 24 & 4 & $-5.34(1)$ & 5.1 & -5.340 & 5.1 & $-5.482(1)$ & 2.6 & -5.403 & 4.0 & -5.626 \\
\hline 24 & 8 & $-2.929(2)$ & 0.8 & -2.860 & 3.2 & $-2.931(1)$ & 0.7 & -2.900 & 1.8 & -2.953 \\
\hline 36 & 0 & $-21.82(1)$ & 4.6 & -22.035 & 3.6 & $-22.21(1)$ & 2.8 & -22.421 & 1.9 & -22.860 \\
\hline 36 & 4 & $-7.93(3)$ & 6.0 & -8.127 & 3.7 & $-8.17(1)$ & 3.2 & -8.173 & 3.2 & -8.440 \\
\hline 36 & 8 & $-4.390(2)$ & 0.9 & -4.302 & 2.9 & $-4.400(1)$ & 0.7 & -4.355 & 1.7 & -4.430 \\
\hline \multicolumn{11}{|c|}{ Open Boundary Conditions } \\
\hline 12 & 0 & $-7.204(1)$ & 1.3 & -7.185 & 1.5 & $-7.274(1)$ & 0.3 & -7.265 & 0.4 & -7.296 \\
\hline 12 & 4 & $-3.748(1)$ & 4.0 & -3.787 & 3.0 & $-3.887(1)$ & 0.5 & -3.894 & 0.3 & -3.905 \\
\hline 12 & 8 & $-2.847(2)$ & 4.6 & -2.920 & 2.2 & $-2.971(1)$ & 0.4 & -2.981 & 0.1 & -2.984 \\
\hline 24 & 0 & $-14.593(1)$ & 2.2 & -14.609 & 2.1 & $-14.767(1)$ & 1.1 & -14.838 & 0.6 & -14.926 \\
\hline 24 & 4 & $-6.32(1)$ & 7.8 & -6.543 & 4.5 & $-6.687(1)$ & 2.4 & -6.803 & 0.7 & -6.851 \\
\hline 24 & 8 & $-4.287(2)$ & 6.6 & -4.414 & 3.8 & $-4.498(2)$ & 2.0 & -4.576 & 0.3 & -4.590 \\
\hline 36 & 0 & $-21.978(2)$ & 2.6 & -22.035 & 2.3 & $-22.260(2)$ & 1.3 & -22.421 & 0.6 & -22.562 \\
\hline 36 & 4 & $-8.83(3)$ & 9.1 & -9.323 & 4.0 & $-9.36(1)$ & 3.6 & -9.625 & 0.9 & -9.713 \\
\hline 36 & 8 & $-5.660(2)$ & 7.3 & -5.873 & 3.8 & $-5.934(3)$ & 2.8 & -6.078 & 0.4 & -6.104 \\
\hline \multicolumn{2}{|c|}{ dof } & 8 & & 18 & & 16 & & 32 & & \\
\hline
\end{tabular}

change in the variational parameters and reject extremely large changes during a single iteration. ${ }^{23}$ For CPS, this can be achieved by adding a dynamically adjusted diagonal shift to $\mathcal{H}$ that penalizes large changes away from $C^{\mu}: \delta \mathcal{H}_{00}=0$, $\delta \mathcal{H}_{i i}>0$. Using this sweep algorithm, we find that the variational energy of the CPS converges (within statistical error) in less than five sweeps.

To obtain the numbers in Tables I and II, we ran the linear-optimization routine for each system through three or four sweeps, after which the energy stopped decreasing and instead fluctuated within a small range of values. We chose one wave function (set of correlators) from the final sweep and calculated the energy and variance reported in the tables using a larger number of Monte Carlo samples than we used during the optimization procedure.

\section{B. Results}

Table I shows the optimized energies obtained for the 2D square Heisenberg model. This model tests the ability of CPS to describe two-dimensional correlations. When open boundary conditions are used, the system is not translation invariant and requires the kind of general parameterization of the CPS emphasized here rather than the more restricted forms used by Huse and Elser. ${ }^{10}$
The nearest-neighbor two-site CPS, CPS[2], has only four variational parameters per site and gives errors in the range of 3-5 $\%$ for open boundary conditions and $1-3 \%$ for periodic boundary conditions. The error is rapidly reduced by increasing the correlator size. For example, for the $8 \times 8$ periodic model, going from pair to $2 \times 2$ to $3 \times 3$ plaquettes, the error goes from $3.1 \%$ to $1.8 \%$ to $0.9 \%$. The rapid convergence of the error with the correlator size is consistent with the results of Mezzacapo et al. ${ }^{13}$ for hardcore boson systems with periodic boundary conditions.

As discussed earlier, CPS with local correlators like those used in Table I satisfy an area law, which allows them to accurately simulate systems with a finite correlation length. However, the 2D Heisenberg model is gapless with longrange correlations, so we expect the error to increase as the size of the lattice increases. Nonetheless, the energetic error of the CPS wave function with a fixed correlator size grows quite slowly as the lattice size is increased. This is not true of MPS, in which the number of variational parameters per site required to achieve a given accuracy grows rapidly with the width of a 2D system.

We performed a series of DMRG calculations for the Heisenberg model on the lattices in Table I with a range of values of $m$ using ALPS. ${ }^{21}$ The variational objects in the DMRG are $m \times m$ matrices. For periodic boundary conditions, $m \approx 35,250$, and 750 are required for 1 percent accu- 
racy on the $4 \times 4,6 \times 6$, and $8 \times 8$ lattices, respectively. The latter calculation, which utilizes about 1.1 million variational parameters per site (neglecting symmetry and conservation of quantum numbers), is to be contrasted with the much more compact description using the CPS with $3 \times 3$ correlators, which corresponds to just 512 parameters per site.

The spinless 1D Hubbard model with periodic boundary conditions has nontrivial fermion correlations and cannot be mapped onto a local spin model. Consequently, this model tests the ability of the CPS to capture fermion correlations. In Table II we compare three-site and four-site nearest-neighbor CPS energies, CPS[3] and CPS[4], with DMRG calculations for $m=3$ and 4 renormalized states. DMRG calculations were carried out using ALPS. ${ }^{21}$ For open boundary conditions, the error in the CPS energy is smallest in the noninteracting system and largest for an intermediate interaction strength $(U=4)$. For periodic boundary conditions, the CPS[4] errors range from less than $1 \%$ for the $U=8$ case to approximately $3 \%$ for the free fermion system-a difficult limit for a locally entangled state. The DMRG energies follow the same trends.

To make a meaningful comparison with the DMRG results, we also show the approximate number of variational degrees of freedom per site in each ansatz. A DMRG $[m]$ wave function has $\mathcal{O}\left(2 m^{2} L\right)$ degrees of freedom (two $m \times m$ matrices at each site) whereas the CPS $[n]$ wave function has $\mathcal{O}\left(2^{n} L\right)$ degrees of freedom (an $n$-site correlator at each site). As a result, the CPS[4] wave function has a similar complexity to the DMRG[3] state. Depending on the boundary conditions and the length of the lattice, the exact number of degrees of freedom may be less than this estimate. For instance, when $L=12$ for an open chain, the DMRG[3] wave function has about 14.7 parameters per site and the CPS[4] wave function has 12. Comparing the CPS and DMRG calculations with similar numbers of variational parameters, we see that the CPS energies are indeed very competitive, especially for periodic boundary conditions, where a CPS includes direct correlations between the ends of the chain.

Minimizing the CPS energy is a nonlinear optimization problem and the sweep algorithm may not converge to the global minimum of the variational energy. We have repeated the optimization for different initial wave functions to avoid local minima. The DMRG algorithm can also converge to a local minimum for $m=3$ or 4 . We repeated each of these DMRG calculations 100 times with the same input and re- ported the lowest energy obtained in Table II. Although convergence to local minima is possible in both CPS and DMRG calculations, we believe the results reported in Tables I and II indicate the competitive accuracy of CPS as a general variational method.

\section{CONCLUSION}

In this paper, we evaluated correlator product states as a route to describing strongly correlated wave functions in any dimension. Our preliminary numerical studies indicate that CPS can capture both nontrivial fermion correlations and two-dimensional correlations. Together with the analysis showing the connections between CPS and many interesting quantum states, this supports the intriguing possibility that CPS are sufficiently flexible to systematically approximate general strongly correlated spin and fermion problems in two or more dimensions.

Nonetheless, many questions remain to be answered. For example, how well do CPS reproduce correlation functions? While properties are harder to obtain accurately than energies in variational calculations, our view is that so long as successive $\operatorname{CPS}[n]$ calculations form a sufficiently rapidly convergent approximation to the quantum state of interest, then accurate approximations to correlation functions can be constructed, as in the case of DMRG calculations. Detailed investigations of such questions and the analysis of more complex systems like the full Hubbard model or the $t-J$ model will require more sophisticated numerical treatments and alternative numerical techniques such as deterministic evaluation methods. We are currently exploring these areas.

\section{ACKNOWLEDGMENTS}

We thank C. L. Henley for bringing the work of Huse and Elser to our attention, T. Nishino for pointing out the long history of CPS, and S. R. White for helpful discussions. This work was supported by the National Science Foundation through Grant No. CHE-0645380 and CHE-1004603, the David and Lucile Packard Foundation, the Alfred P. Sloan Foundation, and the Camille and Henry Dreyfus Foundation. Supplemental funding for C. J. Unrigar was provided through the DOE-CMSN program through Grant No. DE-FG02-07ER46365. *gc238@ cornell.edu

${ }^{1}$ S. R. White, Phys. Rev. Lett. 69, 2863 (1992).

${ }^{2}$ U. Schollwöck, Rev. Mod. Phys. 77, 259 (2005).

${ }^{3}$ A. Gendiar, N. Maeshima, and T. Nishino, Prog. Theor. Phys. 110, 691 (2003).

${ }^{4}$ F. Verstraete, M. M. Wolf, D. Perez-Garcia, and J. I. Cirac, Phys. Rev. Lett. 96, 220601 (2006).

${ }^{5}$ G. Evenbly and G. Vidal, Phys. Rev. Lett. 102, 180406 (2009).

${ }^{6}$ A. W. Sandvik and G. Vidal, Phys. Rev. Lett. 99, 220602 (2007).

${ }^{7}$ H. C. Jiang, Z. Y. Weng, and T. Xiang, Phys. Rev. Lett. 101,
090603 (2008).

${ }^{8}$ Z. C. Gu, M. Levin, B. Swingle, and X. G. Wen, Phys. Rev. B 79, 085118 (2009).

${ }^{9}$ M. P. Nightingale and H. W. J. Blöte, Phys. Rev. B 33, 659 (1986).

${ }^{10}$ D. A. Huse and V. Elser, Phys. Rev. Lett. 60, 2531 (1988).

${ }^{11}$ A. Gendiar and T. Nishino, Phys. Rev. E 65, 046702 (2002).

${ }^{12}$ L. Isaev, G. Ortiz, and J. Dukelsky, Phys. Rev. B 79, 024409 (2009) this study uses correlators which do not overlap and which are treated in a mean-field way. 
${ }^{13}$ F. Mezzacapo, N. Schuch, M. Boninsegni, and J. I. Cirac, New J. Phys. 11, 083026 (2009) the ansatz considered here has the same form as the CPS.

${ }^{14}$ N. Schuch, M. M. Wolf, F. Verstraete, and J. I. Cirac, Phys. Rev. Lett. 100, 040501 (2008).

${ }^{15}$ R. B. Laughlin, Phys. Rev. Lett. 50, 1395 (1983).

${ }^{16}$ A. Yu. Kitaev, Ann. Phys. 303, 2 (2003).

${ }^{17}$ M. M. Wolf, F. Verstraete, M. B. Hastings, and J. I. Cirac, Phys. Rev. Lett. 100, 070502 (2008).

${ }^{18}$ S. Liang, B. Doucot, and P. W. Anderson, Phys. Rev. Lett. 61, 365 (1988).

${ }^{19}$ A. Parola, F. Becca, L. Capriotti, and S. Sorella, J. Magn. Magn. Mater. 272, 138 (2004).
${ }^{20}$ G. K.-L. Chan (unpublished).

${ }^{21}$ A. Albuquerque, F. Alet, P. Corboz, P. Dayal, A. Feiguin, S. Fuchs, L. Gamper, E. Gull, S. Gürtler, A. Honecker, R. Igarashi, M. Körner, A. Kozhevnikov, A. Läuchli, S. R. Manmana, M. Matsumoto, I. P. McCulloch, F. Michel, R. M. Noack, G. Pawłowski, L. Pollet, T. Pruschke, U. Schollwöck, S. Todo, S. Trebst, M. Troyer, P. Werner, S. Wessel, and the ALPS collaboration, J. Magn. Magn. Mater. 310, 1187 (2007).

${ }^{22}$ M. P. Nightingale and V. Melik-Alaverdian, Phys. Rev. Lett. 87, 043401 (2001)

${ }^{23}$ J. Toulouse and C. J. Umrigar, J. Chem. Phys. 126, 084102 (2007). 